\title{
The $C$-Terminus of Hepatitis B Virus-encoded X Protein Is Required for Lapatinib Sensitivity in Hepatocellular Carcinoma Cells
}

\author{
JHEN-YU CHEN ${ }^{1,2^{*}}$, WEI-CHIEN HUANG ${ }^{1,2,3,4,5,6^{*}}$, CHING-TING WEI $^{7,8}$, \\ PEI-HSUAN CHIEN ${ }^{9}$ and YUN-JU CHEN $7,9,10$ \\ ${ }^{1}$ Graduate Institute of Cancer Biology, China Medical University, Taichung, Taiwan, R.O.C.; \\ ${ }^{2}$ The Ph.D. Program for Cancer Biology and Drug Discovery, \\ China Medical University and Academia Sinica, Taichung, Taiwan, R.O.C.; \\ ${ }^{3}$ Graduate Institute of Biomedical Science, China Medical University, Taichung, Taiwan, R.O.C.; \\ ${ }^{4}$ Center for Molecular Medicine, China Medical University and Hospital, Taichung, Taiwan, R.O.C.; \\ ${ }^{5}$ Department of Biotechnology, College of Health Science, Asia University, Taichung, Taiwan, R.O.C.; \\ ${ }^{6}$ Research Center for New Drug Development, China Medical University, Taichung, Taiwan, R.O.C.; \\ ${ }^{7}$ School of Medicine for International Students, I-Shou University, Kaohsiung, Taiwan, R.O.C.; \\ ${ }^{8}$ Division of General Surgery, Department of Surgery, E-Da Hospital, Kaohsiung, Taiwan, R.O.C.; \\ ${ }^{9}$ Department of Medical Research, E-Da Hospital, Kaohsiung, Taiwan, R.O.C.; \\ ${ }^{10}$ Department of Pharmacy, E-Da Hospital, Kaohsiung, Taiwan, R.O.C.
}

\begin{abstract}
Background/Aim: Hepatitis B virus-encoded X protein $(H B x)$ plays a pivotal role in hepatocellular carcinoma (HCC) progression and treatment resistance. Interestingly, our previous study unexpectedly showed that full-length $H B x$ sensitized HCC cells to lapatinib by up-regulating erb-b2 receptor tyrosine kinase 3 (ERBB3). We further aimed to map the exact motif within the $H B x$ sequence responsible for lapatinib sensitization. Materials and Methods: The exact motif responsible for the lapatinib sensitization was assessed by construction of various fragments of $H B x$. Cell viability was examined by the MTT assay and crystal violet staining. Results: Our investigation found that lapatinib sensitivity and up-regulation of ERBB3 promoter activity were observed only in $H C C$ cells expressing $C$-terminal residues of $H B x$. Furthermore, $C$-terminal HBx peptide induced ERBB3 protein expression and sensitivity to lapatinib. Conclusion: These results not only indicate that the $C$-terminus of $H B x$ is required for lapatinib sensitivity, but also provide clues to
\end{abstract}

\footnotetext{
*These Authors contributed equally to this study.

Correspondence to: Yun-Ju Chen, School of Medicine for International Students, I-Shou University, No. 8, Yida Rd., Jiaosu Village, Yanchao District, Kaohsiung City 82445, Taiwan, R.O.C. E-mail: yjchen0326@isu.edu.tw
}

Key Words: HCC, $\mathrm{HBx}$, lapatinib. developing a predictive biomarker for response of $\mathrm{HCC}$ to lapatinib in the future.

To date, hepatocellular carcinoma (HCC) remains a cancer type with high mortality rate due to its heterogeneity and complication. Among the contributing factors for HCC, viral infection is responsible for most cases of HCC and hepatitis B virus $(\mathrm{HBV})$ is the dominant type $(1,2)$. Therefore, many efforts are being made to identify the molecular mechanisms underlying $\mathrm{HBV}$-associated HCC. Accumulating evidence has indicated that $\mathrm{HBV}$-encoded $\mathrm{X}$ protein $(\mathrm{HBx})$, an $\mathrm{HBV}$ encoded regulatory protein in the HBV genome, has the most significant contribution to the carcinogenesis of HBVassociated $\mathrm{HCC}$ (3). $\mathrm{HBx}$ is a multifaceted oncogenic regulator, making it able to function as an activator of signaling pathways in the cytoplasm and as a transcription factor in the nucleus to regulate gene expression and in turn enable tumor survival, proliferation, and metastasis $(4,5)$. In addition to the contributory role in $\mathrm{HBV}$-associated $\mathrm{HCC}$ tumorigenesis, $\mathrm{HBx}$ is reported to be involved in multidrug resistance to chemotherapy. The exact mechanism underlying resistance is not yet completely clarified. HBx-mediated anti-apoptotic effect is now thought to be one underlying mechanism (6-9). Interestingly, our previous study noted that full-length HBx upregulated nuclear factor-kB-dependent erb-b2 receptor tyrosine kinase 3 (ERBB3) expression at the transcriptional level. Furthermore, HBx itself interacts with ERBB2 and ERBB3 proteins, and in turn facilitates the formation of ERBB2- 
ERBB3 heterodimeric complex. This led to sensitization of HCC cells to the ERBB2 tyrosine kinase inhibitor lapatinib (10). Several lines of evidence have revealed that random integration of $\mathrm{HBV}$ into the host genome is commonly detected in most $\mathrm{HBV}$-associated HCC cases, which subsequently results in the truncation of the $\mathrm{HBV}$ genome, especially at the $C$-terminus of $\mathrm{HBx}$ gene locus $(11,12)$. The $\mathrm{HBx}$ sequence is roughly divided into three regions, including $\mathrm{N}$ ( $N$-terminus), $\mathrm{M}$ (middle region) and $\mathrm{C}$ ( $C$-terminus) regions. In this study, we aimed to map the exact motif within the $\mathrm{HBx}$ sequence responsible for lapatinib sensitivity in HCC cells.

\section{Materials and Methods}

Cell lines and reagents. Hep3B HCC cell line from the American Type Culture Collection (Manassas, VA, USA) was maintained in Dulbecco's modified Eagle's medium (DMEM)/nutrient mixture F12 supplemented with $10 \%$ fetal bovine serum (Thermo Fisher Scientific, Waltham, MA, USA). Huh7 HCC cell line from the Japanese Collection of Research Bioresources (Tokyo, Japan) was maintained in DMEM supplemented with $1 \%$ L-glutamine as well as $10 \%$ fetal bovine serum (Thermo Fisher Scientific). Antibody against ERBB3 was purchased from Santa Cruz Biotechnology (Santa Cruz, CA, USA). Anti-HBx was from Abcam (Cambridge, UK) or GeneTex (Irvine, CA, USA). Antibody against actin, dimethyl sulfoxide (DMSO) and 3-(4,5-dimethylthiazol-2-yl)-2,5diphenyltetrazolium bromide (MTT) were purchased from SigmaAldrich (St. Louis, MO, USA). Transfection reagent TransIT-2020 was from Mirus Bio LLC (Madison, WI, USA).

Synthesis and treatment of the trans-activator of transcription (TAT)HBx peptides. To make sure the peptide was able to enter cells, a cell-penetrating peptide, TAT peptide (GRKKRRQRRRPQ) derived from TAT of human immunodeficiency virus attached to $\mathrm{HBx}-C$ terminal peptide (amino acids 103-154) at the $\mathrm{N}$-terminus was synthesized and purchased from (New Taipei City, Taiwan, ROC). HCC cells were treated with $0.1 \mu \mathrm{M}$ TAT-HBx-C (103-154) peptide or control scramble peptide for 1 hour, followed by individual treatments with vehicle control (DMSO), $0.5,1,5 \mu \mathrm{M}$ lapatinib for 3 days, followed by viability assay. On the other hand, HCC cells were treated with vehicle control (DMSO) or $0.1,1,10 \mu \mathrm{M}$ TAT-HBx-C (103-154) peptide for 2 days, followed by western blot.

Construction of hemagglutinin (HA)-HBx fragment expression vector. $\mathrm{HBx}$ fragments, including bp 1-165 (N), bp 1-306 (N+M) and bp 307-462 (C) were amplified by polymerase chain reaction (PCR) using pcDNA-6A-myc-HBx as template as well as primers containing EcoRI cutting site at the 5 '-end and Xhol cutting site at the 3 '-end. The PCR conditions were as follows: denaturation at $95^{\circ} \mathrm{C}$ for 30 seconds, annealing at $52^{\circ} \mathrm{C}$ for $30 \mathrm{sec}$, and extension at $72^{\circ} \mathrm{C}$ for $1 \mathrm{~min}$ for 30 cycles. The PCR products of HBx fragment were purified by gel extraction. The $\mathrm{HBx}$ fragments were constructed into self-made pcDNA-6A-HA expression vector by using the EcoRI and XhoI cutting sites. The HBx fragments and pcDNA-6A-HA expression vector were digested with EcoRI and $\mathrm{XhoI}$ at $37^{\circ} \mathrm{C}$ overnight. The digested HBx fragments and pcDNA6A-HA expression vector were ligated and transformed. The sequences of the HA-HBx fragment were confirmed by sequencing.
Transfection assay. HCC cells with $70-80 \%$ confluence were transfected with $1 \mu \mathrm{g}$ DNA of HA-HBx fragment or pcDNA-6AHA empty vector in 6-cm dish using TransIT-2020 transfection reagent at $1: 1$ ratio. One day later, cells at a density of $1 \times 10^{5}$ cells/well were re-seeded in 6-well plates and used in subsequent experiments.

Cell viability assay. In vitro cell viability assay was carried out either by using MTT colorimetric assay, crystal violet staining or bright-field imaging. For MTT assay, HCC cells at a density of $5 \times 10^{3}$ cells/well were seeded in 96-well plates overnight. Cells were then treated with $0.1 \mu \mathrm{M}$ TAT-HBx-C (103-154) peptide or control scramble peptide for $1 \mathrm{~h}$, followed by individual treatments with vehicle control (DMSO), $0.5,1$, or $5 \mu \mathrm{M}$ lapatinib. Three days later, relative cell numbers were determined by adding $1 \mu \mathrm{g} / \mathrm{ml}$ MTT to each well then incubating for 4 hours. The resulting formazan was solubilized in $100 \mu \mathrm{l}$ DMSO per well and the absorbance was measured at $570 \mathrm{~nm}$. For crystal violet staining assay and brightfield imaging, HA-HBx fragment-transfected HCC cells at a density of $1 \times 10^{5}$ cells/well were seeded in 6 -well plates overnight, followed by treatment with vehicle control (DMSO) or $1 \mu \mathrm{M}$ lapatinib for 3 days. Relative cell numbers were determined by $1 \%$ crystal violet staining. In brief, cells were washed with $1 \times$ phosphate-buffered saline twice and fixed, followed by staining with $1 \%$ crystal violet dissolved in $30 \%$ ethanol for $30 \mathrm{~min}$ at room temperature. Next, cells were washed with tap water twice in order to eliminate background interference. The crystal violet-stained plates were air dried and subjected to photography. In addition to determination by $1 \%$ crystal violet staining, the relative cell numbers were examined by bright-field imaging under microscopy.

Reporter assay. HCC cells at a density of $3 \times 10^{4}$ cells/well were seeded in 12-well plates overnight. ERBB3 promoter luciferase DNA and HA-HBx fragment DNA as well as Renilla luciferase DNA were co-transfected into cells for 2 days, followed by cell lysis. The luciferase activity was determined by using SecretePair $^{\text {TM }}$ Gaussia Luciferase Assay Kit (GeneCopoeia, Rockville, MD, USA) based on the manufacturer's protocol. In brief, $10 \mu \mathrm{l}$ whole cell lysates were mixed with $100 \mu \mathrm{l} 1 \times$ assay working solution containing luminescent substrate in a luminometer tube and the luciferase activity was measured by luminometer. Firefly luciferase activities were normalized with the renilla activities. The experiment was independently performed three times.

Western blot. HCC cells were treated with vehicle control (DMSO) or $0.1,1$, or $10 \mu \mathrm{M}$ TAT-HBx-C (103-154) peptide for 2 days. The whole cell lysates were harvested, and subjected to western blot procedure, including electrophoresis, gel transfer and blocking. Protein expression was examined using antibodies against ERBB3 $(1: 1,000), \operatorname{HBx}(1: 1,000)$ and actin $(1: 10,000)$

Statistical analysis. The statistical analysis was performed using Student's $t$-test. $p$-Values of less than 0.05 were considered statistically significant.

\section{Results}

$C$-Terminal residues of $H B x$ were required for lapatinibinduced inhibition of viability and ERBB3 promoter activity in Hep3B HCC cells. To map the exact region within the 
A

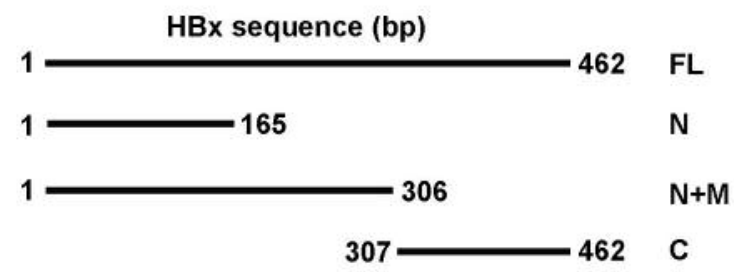

B

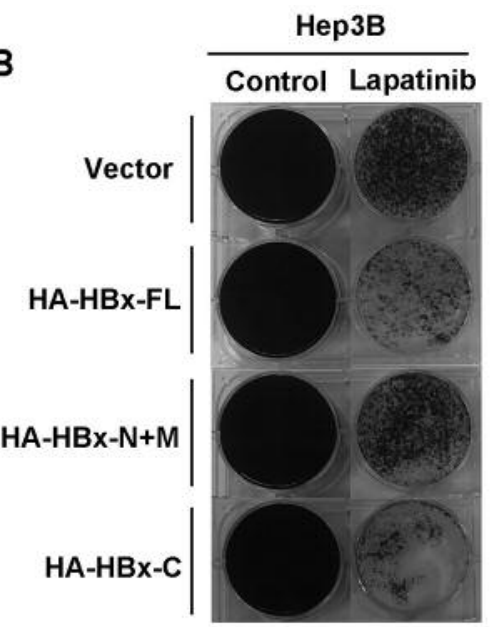

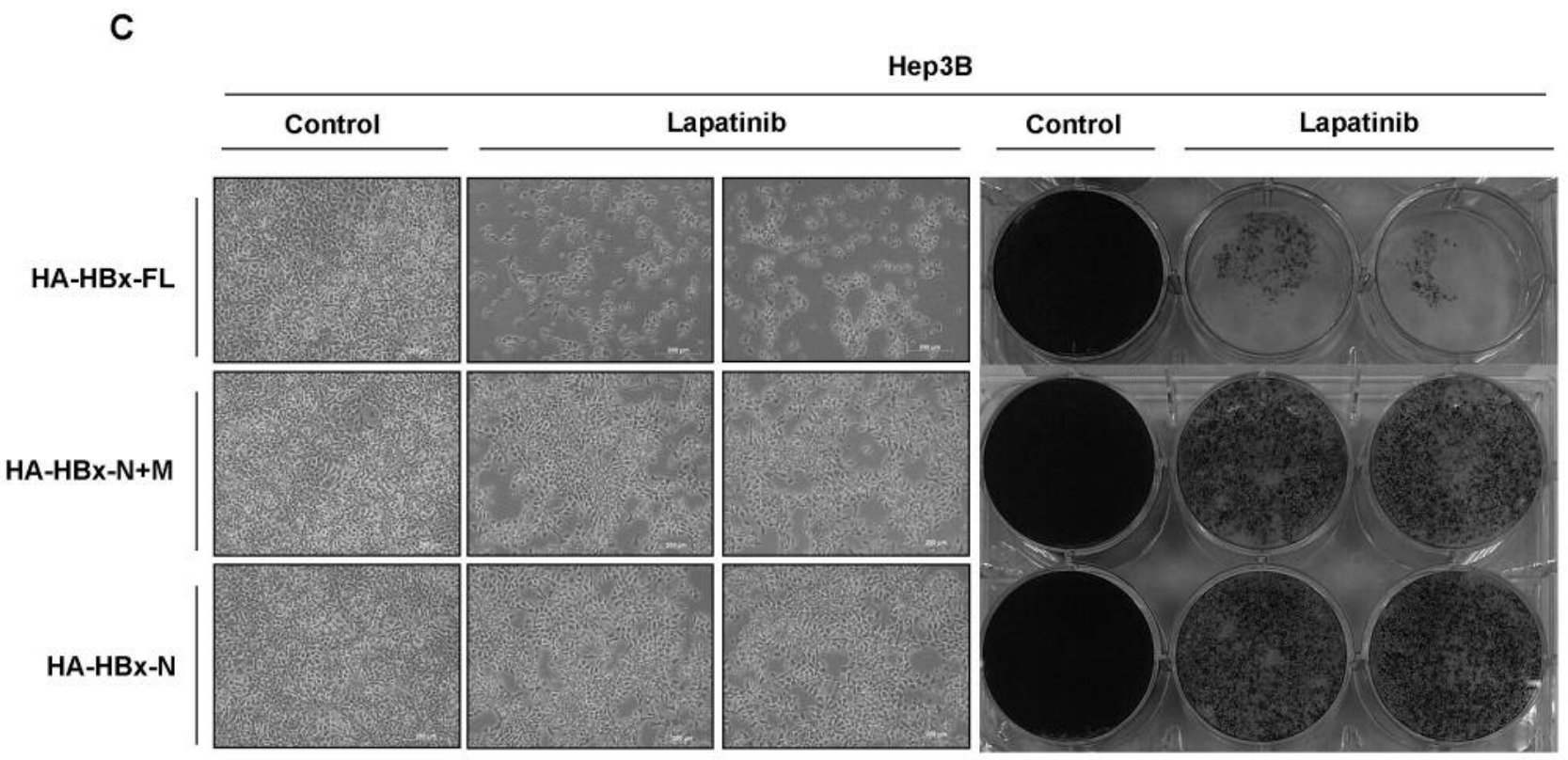

Figure 1. C-Terminal residues of hepatitis B virus-encoded $X$ protein $(H B x)$ were required for lapatinib-induced viability inhibition in Hep3B hepatocellular carcinoma (HCC) cells. A: The illustration of different HBx fragments. $B$ and C: Individual hemagglutinin-HBx fragment expression vectors were transfected into Hep $3 B$ HCC cells, followed by $1 \mu \mathrm{M}$ lapatinib treatment for 3 days. Cell viability was determined by crystal violet staining or bright-field imaging under microscopy. FL: full-length HBx, bp 1-462; N: N-terminus of HBx, bp 1-165; N+M: N-terminus and middle region of HBx, bp 1-306; C: C-terminus of HBx, bp 307-462.

HBx sequence responsible for lapatinib sensitivity in HCC cells, several $\mathrm{HBx}$ fragments were designed and constructed into expression vectors. The $\mathrm{HBx}$ fragments containing full length (FL), $\mathrm{N}, \mathrm{N}+\mathrm{M}$ and $\mathrm{C}$ regions were constructed into HA-tagged expression vector (Figure 1A). These HBx fragment expression vectors were transfected into Hep3B HCC cells, followed by lapatinib treatment. As shown in Figure 1B, lapatinib induced apparent inhibition of viability in Hep3B cells with HA-HBx-FL expression as compared to that in the group with empty vector. However, such inhibitory effect was no longer observed in Hep3B cells with
HA-HBx-N+M expression. Lapatinib induced similar inhibition of viability in HA-HBx-C-expressing Hep3B cells as that in HA-HBx-FL-expressing ones. In support of this observation, the results in Figure 1C show that neither Hep3B cells expressing $\mathrm{HBx}-\mathrm{N}+\mathrm{M}$ nor those expressing HA-HBx-N displayed much more inhibition of viability by lapatinib than did cells expressing HA-HBx-FL.

Since our previous study indicated that $\mathrm{HBx}$ sensitizes HCC cells to lapatinib by up-regulating $E R B B 3$ transcription (10), we further examined the effects of $\mathrm{HBx}$ fragments on ERBB3 promoter activity. As shown in Figure 2, only 
HBx-FL and HBx-C fragments significantly increased $E R B B 3$ promoter activity. Collectively, these results suggest that the $C$-terminal residue of $\mathrm{HBx}$ are required for lapatinibinduced viability inhibition and $E R B B 3$ promoter activity in HCC cells.

HBx C-terminal peptide induced ERBB3 expression and lapatinib sensitivity in HCC cells. Next, we were interested in whether the $C$-terminus of $\mathrm{HBx}$ acts as a sensitizer for lapatinib treatment. To this end, TAT-HBx peptide containing $C$-terminal region amino acids 103-154 was synthesized. Hep3B and Huh7 HCC cells were treated with different concentrations of TAT-HBx-C peptide for 2 days and ERBB3 protein expression was examined. The results showed that TAT-HBX-C peptide significantly induced ERBB3 protein expression in both Hep3B and Huh7 HCC cells (Figure 3A). We further examined the effects of TAT-HBx-C peptide on sensitivity to lapatinib. Huh7 cells were pre-treated with TAT-HBx-C peptide or scramble control peptide, followed by different concentrations of lapatinib. As shown in Figure 3B, lapatinib induced greater dose-dependent inhibition of viability in cells pre-treated with TAT-HBx-C peptide than in the scramble control group. Taken together, these results imply HBx $C$-terminal peptide acts as a sensitizer to enhance lapatinib sensitivity in HCC cells.

\section{Discussion}

It is well known that virus infection is responsible for most cases of HCC and HBV is the dominant type. Therefore, fully understanding the mechanisms underlying $\mathrm{HBV}$-associated $\mathrm{HCC}$ is of great significance (13). Among these, HBx, the smallest protein in $\mathrm{HBV}$ genome with 154 amino acids has the greatest contribution to tumorigenesis in HCC. It is reported that random $\mathrm{HBV}$ integration is detected in most host genomes from $\mathrm{HBV}$-infected HCC cases and the HBx sequence is a preferential site of integration into the human genome, resulting in truncation of $\mathrm{HBx}$ gene locus at the $C$-terminus and in turn facilitating $\mathrm{HCC}$ development in chronically infected patients $(12,14-16)$. The $C$-terminus of $\mathrm{HBx}$ is reportedly associated with an inhibitory role in cell proliferation, since overexpression of $C$-terminal-truncated $\mathrm{HBx}$ was shown to lead to cell growth of HCC $(17,18)$. Similarly, overexpression of centromere protein $\mathrm{A}$, a protein required for chromosome segregation in mitosis, has been found to be closely associated with $C$-terminal mutation of HBx in HCC (19). Furthermore, two recent studies indicate that $C$-terminal-truncated $\mathrm{HBx}$ leads to a significant increase in the ability of cell self-renew, resistance to 5-fluorouracil chemotherapy and sorafenib therapy $(9,20)$. However, the comprehensive effects of $C$ terminus-truncated $\mathrm{HBx}$ on HCC remains unclear.

In this study, we found that sensitivity to lapatinib and upregulation of $E R B B 3$ promoter activity were observed only

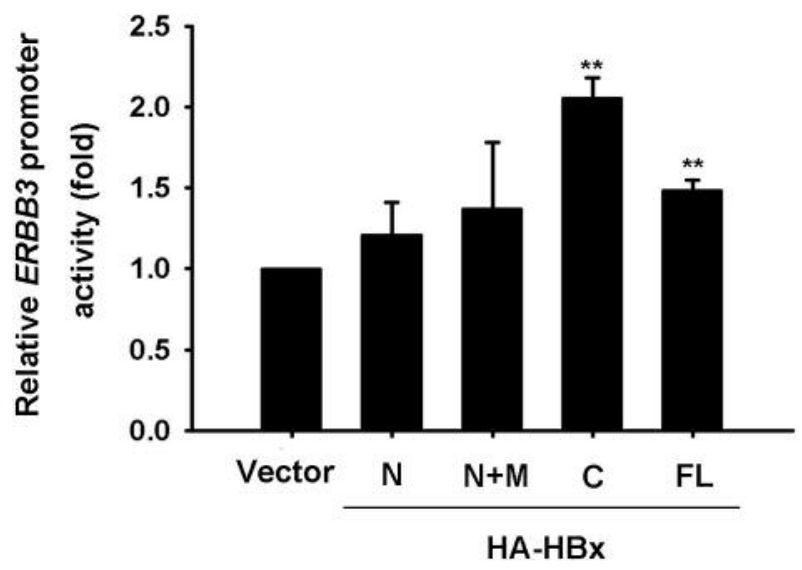

Figure 2. $C$-Terminal residues of hepatitis $B$ virus-encoded $X$ protein $(H B x)$ were required for erb-b2 receptor tyrosine kinase 3 (ERBB3) promoter activity in Hep3B hepatocellular carcinoma cells. ERBB3 promoter luciferase DNA and different hemagglutinin-HBx fragment DNA as well as Renilla luciferase DNA were co-transfected into Hep3B $H C C$ cells for 2 days. The luciferase activity of ERBB3 promoter was determined using Secrete-PairTM Gaussia Luciferase Assay Kit. Firefly luciferase activities were normalized using Renilla activities. Data are means $\pm S D$ of three determinations. FL: Full-length HBx, bp 1-462; N: $N$-terminus of $H B x$, bp $1-165 ; N+M: N$-terminus and middle region of $H B x$, bp 1-306; C: C-terminus of HBx, bp 307-462. **Significantly different at $p<0.01$ as compared to the control (vector) group by Student's t-test.

in HCC cells with HBx $C$-terminal residues. Furthermore, $C$ terminal HBx peptide induced ERBB3 protein expression and lapatinib sensitivity. As far as we are aware, our study is the first to report that the $C$-terminus of $\mathrm{HBx}$ is required for sensitivity of HCC cells to lapatinib. Most important of all, our study, as well as two recent studies $(9,20)$, consistently suggest $C$-terminal residues of $\mathrm{HBx}$ to be a predictive biomarker for the efficacy of chemotherapy and targeted therapy in HCC, which awaits further validation in the clinic. On the other hand, our study also shows that pretreatment with $C$-terminal $\mathrm{HBx}$ peptide enhanced the sensitivity of HCC cells to lapatinib. Since it is reported that an increase in tumor incidence and size was observed in cells expressing $C$-terminus truncated $\mathrm{HBx}$ as compared to cells expressing FL HBx (9), it implies that the $C$-terminus is not critical for $\mathrm{HBx}$-mediated $\mathrm{HCC}$ tumorigenesis. Therefore, it may be safe to use $C$-terminal $\mathrm{HBx}$ peptide as a sensitizer to enhance the efficacy of lapatinib in HCC treatment, a notion which deserves further investigation.

\section{Conflicts of Interest}

All Authors declare that they have no conflicts of interest in regard to this study. 
A

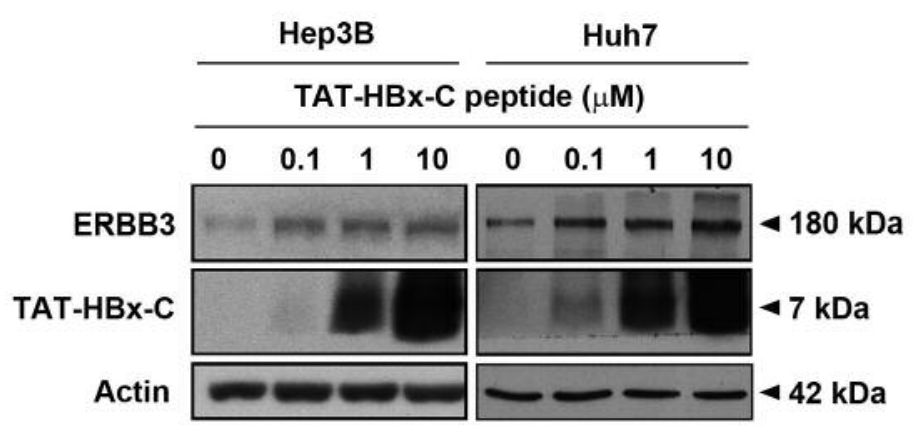

B

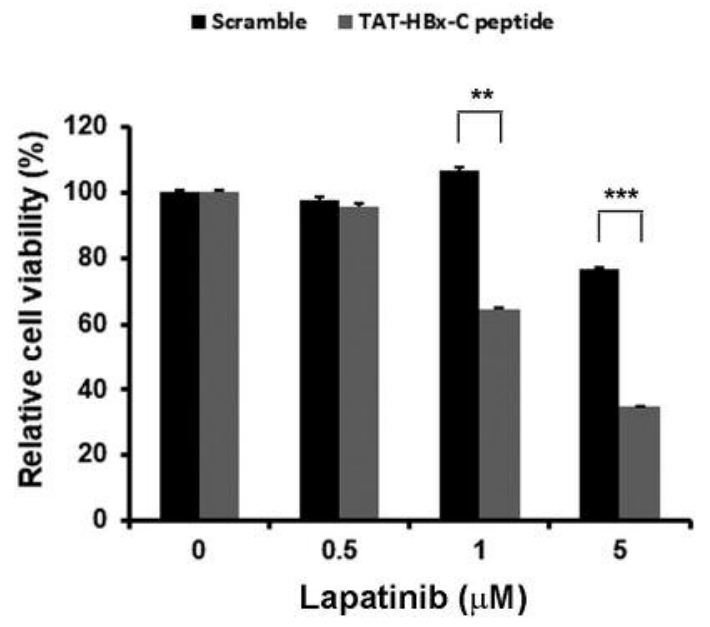

Figure 3. Hepatitis B virus-encoded X protein (HBx) C-terminal peptide induced erb-b2 receptor tyrosine kinase 3 (ERBB3) expression and sensitivity to lapatinib in hepatocellular carcinoma (HCC) cells. A: Hep3B and Huh7 HCC cells were treated with different concentrations of the transactivator of transcription (TAT)-HBx-C (103-154) peptide for 2 days and then whole cell lysates were harvested and subjected to western blot. Protein expressions were examined using antibodies against ERBB3, HBx and actin. B: Huh7 cells were treated with 0.1 $\mu M$ TAT-HBx-C (103-154) peptide or control scramble peptide, followed by treatment with different concentrations of lapatinib for 3 days. Cell viability was then determined by MTT assay. Data are means $\pm S D$ of three determinations. Significantly different at $* * p<0.01$, and $* * * p<0.001$ by Student's t-test.

\section{Funding Sources}

This work was supported by grants from the Ministry of Science and Technology, R.O.C. (MOST 105-2320-B-214-005), E-Da Hospital (EDPJ105083), and China Medical University Hospital (DMR-107-029).

\section{References}

1 Ghouri YA, Mian I and Rowe JH: Review of hepatocellular carcinoma: Epidemiology, etiology, and carcinogenesis. J Carcinog 16: 1, 2017.

2 El-Serag HB: Epidemiology of viral hepatitis and hepatocellular carcinoma. Gastroenterology 142(6): 1264-1273 e1261, 2012.

3 Levrero M and Zucman-Rossi J: Mechanisms of HBV-induced hepatocellular carcinoma. J Hepatol 64(1 Suppl): S84-S101, 2016.

4 Koike K: Hepatitis B virus HBx gene and hepatocarcinogenesis. Intervirology 38(3-4): 134-142, 1995.

5 Tang H, Oishi N, Kaneko S and Murakami S: Molecular functions and biological roles of hepatitis B virus X protein. Cancer Sci 97(10): 977-983, 2006.

6 Chao CC: Inhibition of apoptosis by oncogenic hepatitis B virus $\mathrm{X}$ protein: Implications for the treatment of hepatocellular carcinoma. World J Hepatol 8(25): 1061-1066, 2016.

7 Liu Y, Lou G, Wu W, Zheng M, Shi Y, Zhao D and Chen Z: Involvement of the NF-kappaB pathway in multidrug resistance induced by HBx in a hepatoma cell line. J Viral Hepat 18(10): e439-446, 2011

8 Guan J, Chen XP, Zhu H, Luo SF, Cao B and Ding L: Involvement of extracellular signal-regulated kinase/mitogen- activated protein kinase pathway in multidrug resistance induced by HBx in hepatoma cell line. World J Gastroenterol 10(23): 3522-3527, 2004.

9 Ching RHH, Sze KMF, Lau EYT, Chiu YT, Lee JMF, Ng IOL and Lee TKW: $C$-terminal truncated hepatitis B virus $\mathrm{X}$ protein regulates tumorigenicity, self-renewal and drug resistance via STAT3/NANOG signaling pathway. Oncotarget 8(14): 2350723516, 2017.

10 Chen JY, Chen YJ, Yen CJ, Chen WS and Huang WC: HBx sensitizes hepatocellular carcinoma cells to lapatinib by upregulating ERBB3. Oncotarget 7(1): 473-489, 2016.

11 Wang D, Cai H, Yu WB and Yu L: Identification of hepatitis B virus $\mathrm{X}$ gene variants between hepatocellular carcinoma tissues and pericarcinoma liver tissues in eastern China. Int J Clin Exp Pathol 7(9): 5988-5996, 2014.

12 Tu H, Bonura C, Giannini C, Mouly H, Soussan P, Kew M, Paterlini-Brechot $\mathrm{P}$, Brechot $\mathrm{C}$ and Kremsdorf D: Biological impact of natural $\mathrm{COOH}$-terminal deletions of hepatitis $\mathrm{B}$ virus $\mathrm{X}$ protein in hepatocellular carcinoma tissues. Cancer Res 61(21): 7803-7810, 2001.

13 Raza SA, Clifford GM and Franceschi S: Worldwide variation in the relative importance of hepatitis $\mathrm{B}$ and hepatitis $\mathrm{C}$ viruses in hepatocellular carcinoma: A systematic review. Br J Cancer 96(7): 1127-1134, 2007.

14 Wang Y, Wu MC, Sham JS, Tai LS, Fang Y, Wu WQ, Xie D and Guan XY: Different expression of hepatitis B surface antigen between hepatocellular carcinoma and its surrounding liver tissue, studied using a tissue microarray. J Pathol 197(5): 610616, 2002.

15 Wang Y, Lau SH, Sham JS, Wu MC, Wang T and Guan XY: Characterization of $\mathrm{HBV}$ integrants in 14 hepatocellular 
carcinomas: Association of truncated $\mathrm{X}$ gene and hepatocellular carcinogenesis. Oncogene 23(1): 142-148, 2004.

16 Quetier I, Brezillon N, Revaud J, Ahodantin J, DaSilva L, Soussan P and Kremsdorf D: $C$-terminal-truncated hepatitis B virus $\mathrm{X}$ protein enhances the development of diethylnitrosamineinduced hepatocellular carcinogenesis. J Gen Virol 96(Pt 3): 614-625, 2015.

17 Fung J, Lai CL and Yuen MF: Hepatitis B and C virus-related carcinogenesis. Clin Microbiol Infect 15(11): 964-970, 2009.

18 Ma NF, Lau SH, Hu L, Xie D, Wu J, Yang J, Wang Y, Wu MC, Fung J, Bai X, Tzang CH, Fu L, Yang M, Su YA and Guan XY: COOH-terminal truncated HBV $\mathrm{X}$ protein plays key role in hepatocarcinogenesis. Clin Cancer Res 14(16): 5061-5068, 2008.
19 Liu L, Li Y, Zhang S, Yu D and Zhu M: Hepatitis B virus X protein mutant upregulates CENP-A expression in hepatoma cells. Oncol Rep 27(1): 168-173, 2012.

$20 \mathrm{Ng} \mathrm{KY}$, Chai S, Tong M, Guan XY, Lin CH, Ching YP, Xie D, Cheng AS and Ma S: $C$-terminal truncated hepatitis B virus $\mathrm{X}$ protein promotes hepatocellular carcinogenesis through induction of cancer and stem cell-like properties. Oncotarget 7(17): 24005-24017, 2016.

Received December 10, 2018

Revised December 18, 2018

Accepted December 21, 2018 